\title{
Energy dissipation and scattering angle distribution analysis of the classical trajectory calculations of methane scattering from a $\mathrm{Ni}(111)$ surface
}

\section{Citation for published version (APA):}

Milot, R., Kleyn, A. W., \& Jansen, A. P. J. (2001). Energy dissipation and scattering angle distribution analysis of the classical trajectory calculations of methane scattering from a Ni(111) surface. Journal of Chemical Physics, 115(8), 3888-3894. https://doi.org/10.1063/1.1388224

DOI:

10.1063/1.1388224

Document status and date:

Published: 01/01/2001

\section{Document Version:}

Publisher's PDF, also known as Version of Record (includes final page, issue and volume numbers)

\section{Please check the document version of this publication:}

- A submitted manuscript is the version of the article upon submission and before peer-review. There can be important differences between the submitted version and the official published version of record. People interested in the research are advised to contact the author for the final version of the publication, or visit the $\mathrm{DOI}$ to the publisher's website.

- The final author version and the galley proof are versions of the publication after peer review.

- The final published version features the final layout of the paper including the volume, issue and page numbers.

Link to publication

\footnotetext{
General rights

- You may freely distribute the URL identifying the publication in the public portal. follow below link for the End User Agreement:

www.tue.nl/taverne

Take down policy

If you believe that this document breaches copyright please contact us at:

openaccess@tue.nl

providing details and we will investigate your claim.
}

Copyright and moral rights for the publications made accessible in the public portal are retained by the authors and/or other copyright owners and it is a condition of accessing publications that users recognise and abide by the legal requirements associated with these rights.

- Users may download and print one copy of any publication from the public portal for the purpose of private study or research.

- You may not further distribute the material or use it for any profit-making activity or commercial gain

If the publication is distributed under the terms of Article 25fa of the Dutch Copyright Act, indicated by the "Taverne" license above, please 


\title{
Energy dissipation and scattering angle distribution analysis of the classical trajectory calculations of methane scattering from a Ni(111) surface
}

\author{
Robin Milot \\ Schuit Institute of Catalysis, ST/SKA, Eindhoven University of Technology, P.O. Box 513, NL-5600 MB \\ Eindhoven, The Netherlands
}

\begin{abstract}
A. W. Kleyn
Leiden Institute of Chemistry, Department of Surfaces and Catalysis, Leiden University, P.O. Box 9502, NL-2300 RA Leiden, The Netherlands
\end{abstract}

\author{
A. P. J. Jansen \\ Schuit Institute of Catalysis, ST/SKA, Eindhoven University of Technology, P.O. Box 513, NL-5600 MB \\ Eindhoven, The Netherlands
}

(Received 13 March 2001; accepted 6 June 2001)

\begin{abstract}
We present classical trajectory calculations of the rotational vibrational scattering of a nonrigid methane molecule from a $\mathrm{Ni}(111)$ surface. Energy dissipation and scattering angles have been studied as a function of the translational kinetic energy, the incidence angle, the (rotational) nozzle temperature, and the surface temperature. Scattering angles are somewhat toward the surface for the incidence angles of $30^{\circ}, 45^{\circ}$, and $60^{\circ}$ at a translational energy of $96 \mathrm{~kJ} / \mathrm{mol}$. Energy loss is primarily from the normal component of the translational energy. It is transferred for somewhat more than half to the surface and the rest is transferred mostly to rotational motion. The spread in the change of translational energy has a basis in the spread of the transfer to rotational energy, and can be enhanced by raising of the surface temperature through the transfer process to the surface motion.
\end{abstract}

(C) 2001 American Institute of Physics. [DOI: 10.1063/1.1388224]

\section{INTRODUCTION}

The dissociative adsorption of methane on transition metals is an important reaction in catalysis; it is the rate limiting step in steam reforming to produce syngas, and it is prototypical for catalytic $\mathrm{C}-\mathrm{H}$ activation. Therefore the dissociation is of high interest for many surface scientists. (See, for a recent review, Ref. 1.) Molecular beam experiments in which the dissociation probability was measured as a function of translational energy have observed that the dissociation probability is enhanced by the normal incidence component of the incidence translational energy. ${ }^{2-12}$ This suggests that the reaction occurs primarily through a direct dissociation mechanism at least for high translational kinetic energies. Some experiments have also observed that vibrationally hot $\mathrm{CH}_{4}$ dissociates more readily than cold $\mathrm{CH}_{4}$, with the energy in the internal vibrations being about as effective as the translational energy in inducing dissociation. ${ }^{2-4,7-10,13} \mathrm{~A}$ molecular beam experiment with laser excitation of the $\nu_{3}$ mode did succeed in measuring a strong enhancement of the dissociation on a $\mathrm{Ni}(100)$ surface. However, this enhancement was still much too low to account for the vibrational activation observed in previous studies and indicated that other vibrationally excited modes contribute significantly to the reactivity of thermal samples. ${ }^{14}$

It is very interesting to simulate the dynamics of the dissociation, because of the direct dissociation mechanism, and the role of the internal vibrations. Wave packet simulations of the methane dissociation reaction on transition metals have treated the methane molecule always as a diatomic up to now. ${ }^{15-20}$ Apart from one $\mathrm{C}-\mathrm{H}$ bond (a pseudo $\nu_{3}$ stretch mode) and the molecule surface distance, either (multiple) rotations or some lattice motion were included. None of these studies have looked at the role of the other internal vibrations, so there is no model that describes which vibrationally excited mode might be responsible for the experimental observed vibrational activation.

In previous papers we reported on wave packet simulations to determine which and to what extent internal vibrations are important for the dissociation in the vibrational ground state of $\mathrm{CH}_{4},{ }^{21}$ and $\mathrm{CD}_{4} \cdot{ }^{22}$ We were not able yet to simulate the dissociation including all internal vibrations. Instead we simulated the scattering of methane in fixed orientations, for which all internal vibrations can be included, and used the results to deduce consequences for the dissociation. These simulations indicate that to dissociate methane the interaction of the molecule with the surface should lead to an elongated equilibrium $\mathrm{C}-\mathrm{H}$ bond length close to the surface, and that the scattering was almost elastic. Later on we reported on wave packet simulations of the role of vibrational excitations for the scattering of $\mathrm{CH}_{4}$ and $\mathrm{CD}_{4} \cdot{ }^{23}$ We predicted that initial vibrational excitations of the asymmetrical stretch $\left(\nu_{3}\right)$ but especially the symmetrical stretch $\left(\nu_{1}\right)$ modes will give the highest enhancement of the dissociation probability of methane. Although we have performed these wave packet simulations in ten dimensions, we still had to neglect two translational and three rotational coordinates of the methane molecule and did not account for surface motion and corrugation. It is nowadays still hard to include all these features into a wave packet simulation, therefore we decided to study these with classical trajectory simulations. 
In this article we will present full classical trajectory simulations of methane from a $\mathrm{Ni}(111)$ surface. We are especially interested in the effect of the molecular rotations and surface motion, which we study as a function of the nozzle and surface temperature. The methane molecule is flexible and able to vibrate. We do not include vibrational kinetic energy at the beginning of the simulation, because a study of vibrational excitation due to the nozzle temperature needs a special semi-classical treatment. Besides its relevance for the dissociation reaction of methane on transition metals, our scattering simulation can also be of interest as a reference model for the interpretation of methane scattering itself, which has been studied with molecular beams on $\mathrm{Ag}(111),{ }^{24,25} \mathrm{Pt}(111),{ }^{26-29}$ and $\mathrm{Cu}(111)$ surfaces. ${ }^{30}$ It was observed that the scattering angles are in some cases in disagreement with the outcome of the classical Hard Cube Model (HCM) described in Ref. $31 .{ }^{26,27}$ We will show in this article that the assumptions of this HCM model are too crude for describing the processes obtained from our simulation. The time-of-flight experiments show that there is almost no vibrational excitation during the scattering, ${ }^{28,29}$ which is in agreement with our current classical simulations and our previous wave packet simulations. ${ }^{21,22}$

The rest of this article is organized as follows. We start with a description of our model potential, and an explanation of the simulation conditions. The results and discussion are presented next. We start with the scattering angles, and relate them to the energy dissipation processes. Next we will compare our simulation with other experiments and theoretical models. We end with a summary and some general conclusions.

\section{COMPUTATIONAL DETAILS}

We have used classical molecular dynamics for simulating the scattering of methane from a $\mathrm{Ni}(111)$ surface. The methane molecule was modeled as a flexible molecule. The forces on the carbon, hydrogen, and $\mathrm{Ni}$ atoms are given by the gradient of the model potential energy surface described below. The first-order ordinary differential equations for the Newtonian equations of motion of the Cartesian coordinates were solved with use of a variable-order, variable-step Adams method. ${ }^{32}$ We have simulated at translational energies of $24,48,72$, and $96 \mathrm{~kJ} / \mathrm{mol}$ at normal incidence, and at 96 $\mathrm{kJ} / \mathrm{mol}$ for incidence angles of $30^{\circ}, 45^{\circ}$, and $60^{\circ}$ with the surface normal. The surface temperature and (rotational) nozzle temperature for a certain simulation were taken independently between 200 and $800 \mathrm{~K}$.

\section{A. Potential energy surface}

The model potential energy surface used for the classical dynamics is derived from one of our model potentials with elongated $\mathrm{C}-\mathrm{H}$ bond lengths toward the surface, previously used for wave packet simulation of the vibrational scattering of fixed oriented methane on a flat surface. ${ }^{21,22}$ In this original potential there is one part responsible for the repulsive interaction between the surface and the hydrogens, and another part for the intramolecular interaction between carbon and hydrogens.
We have rewritten the repulsive part in pair potential terms between top layer surface $\mathrm{Ni}$ atoms and hydrogens in such a way that the surface integral over all these $\mathrm{Ni}$ atoms give the same overall exponential fall-off as the original repulsive PES term for a methane molecule far away from the surface in an orientation with three bonds pointing toward the surface. The repulsive pair interaction term $V_{\text {rep }}$ between hydrogen $i$ and $\mathrm{Ni}$ atom $j$ at the surface is then given by

$$
V_{\text {rep }}=\frac{A e^{-\alpha Z_{i j}}}{Z_{i j}},
$$

where $Z_{i j}$ is the distance between hydrogen atom $i$ and $\mathrm{Ni}$ atom $j$.

The intramolecular potential part is split up in bond, bond angle, and cross potential energy terms. The single $\mathrm{C}-\mathrm{H}$ bond energy is given by a Morse function with bond lengthening toward the surface,

$$
V_{\text {bond }}=D_{e}\left[1-e^{-\gamma\left(R_{i}-R_{\mathrm{eq}}\right)}\right]^{2},
$$

where $D_{e}$ is the dissociation energy of methane in the gas phase, and $R_{i}$ is the length of the $\mathrm{C}-\mathrm{H}$ bond $i$. Dissociation is not possible at the surface with this potential term, but the entrance channel for dissociation is mimicked by an elongation of the equilibrium bond length $R_{\mathrm{eq}}$ when the distance between the hydrogen atom and the $\mathrm{Ni}$ atoms in the top layer of the surface become shorter. This is achieved by

$$
R_{\mathrm{eq}}=R_{0}+S \sum_{j} \frac{e^{-\alpha Z_{i j}}}{Z_{i j}},
$$

where $R_{0}$ is the equilibrium $\mathrm{C}-\mathrm{H}$ bond length in the gas phase. The bond elongation factor $S$ was chosen in such a way that the elongation is $0.054 \mathrm{~nm}$ at the classical turning point of $93.2 \mathrm{~kJ} / \mathrm{mol}$ incidence translational energy for a rigid methane molecule, when the molecule approaches a surface $\mathrm{Ni}$ atom atop with one bond pointing toward the surface. The single angle energy is given by the harmonic expression,

$$
V_{\text {angle }}=k_{\theta}\left(\theta_{i j}-\theta_{0}\right)^{2} \text {, }
$$

where $\theta_{i j}$ is the angle between $\mathrm{C}-\mathrm{H}$ bond $i$ and $j$, and $\theta_{0}$ the equilibrium bond angle. Furthermore, there are some crossterm potentials between bonds and angles. The interaction between two bonds are given by

$$
V_{\mathrm{bb}}=k_{R R}\left(R_{i}-R_{0}\right)\left(R_{j}-R_{0}\right) .
$$

The interaction between a bond angle and the bond angle on the other side is given by

$$
V_{\text {aa }}=k_{\theta \theta}\left(\theta_{i j}-\theta_{0}\right)\left(\theta_{k l}-\theta_{0}\right) \text {. }
$$

The interaction between a bond angle and one of its bonds is given by

$$
V_{\mathrm{ab}}=k_{\theta R}\left(\theta_{i j}-\theta_{0}\right)\left(R_{i}-R_{0}\right) .
$$

The parameters of the intramolecular potential energy terms were calculated by fitting the second derivatives of these terms on the experimental vibrational frequencies of $\mathrm{CH}_{4}$ and $\mathrm{CD}_{4}$ in the gas phase. ${ }^{33,34}$

The $\mathrm{Ni}-\mathrm{Ni}$ interaction between nearest-neighbors is given by the harmonic form, 
TABLE I. Parameters of the potential energy surface.

\begin{tabular}{llll}
\hline \hline $\mathrm{Ni}-\mathrm{H}$ & $A$ & 971.3 & $\mathrm{~kJ} \mathrm{~nm} \mathrm{~mol}$ \\
& $\alpha$ & 20.27 & $\mathrm{~nm}^{-1}$ \\
& $S$ & 0.563 & $\mathrm{~nm}^{2}$ \\
$\mathrm{CH}_{4}$ & $\gamma$ & 17.41 & $\mathrm{~nm}^{-1}$ \\
& $D_{e}$ & 480.0 & $\mathrm{~kJ} \mathrm{~mol}$ \\
& $R_{0}$ & 0.115 & $\mathrm{~nm}$ \\
& $k_{\theta}$ & 178.6 & $\mathrm{~kJ} \mathrm{~mol}$ \\
& $\theta_{0}$ & 1.911 & $\mathrm{rad}^{-2}$ \\
& $k_{R R}$ & 4380 & $\mathrm{~kJ} \mathrm{~mol}-1$ \\
& $k_{\theta \theta}$ & 11.45 & $\mathrm{~kJ} \mathrm{~mol}^{-1} \mathrm{rad}^{-2}$ \\
& $k_{\theta R}$ & -472.7 & $\mathrm{~kJ} \mathrm{~mol}^{-1} \mathrm{rad}^{-1} \mathrm{~nm}^{-1}$ \\
$\mathrm{Ni}-\mathrm{Ni}$ & $\lambda_{n n}$ & 28328 & $\mathrm{~kJ} \mathrm{~mol}^{-1} \mathrm{~nm}^{-2}$ \\
& $\mu_{n n}$ & -820 & $\mathrm{~kJ} \mathrm{~mol} \mathrm{~nm}^{-2}$ \\
\hline \hline
\end{tabular}

$$
\begin{aligned}
V_{\mathrm{Ni}-\mathrm{Ni}}= & \frac{1}{2} \lambda_{i j}\left[\left(\mathbf{u}_{\mathbf{i}}-\mathbf{u}_{\mathbf{j}}\right) \cdot \hat{\mathbf{r}}_{\mathbf{i j}}\right] \\
& +\frac{1}{2} \mu_{i j}\left\{\left(\mathbf{u}_{\mathbf{i}}-\mathbf{u}_{\mathbf{j}}\right)^{\mathbf{2}}-\left[\left(\mathbf{u}_{\mathbf{i}}-\mathbf{u}_{\mathbf{j}}\right) \cdot \hat{\mathbf{r}}_{\mathbf{i j}}\right]^{\mathbf{2}}\right\} .
\end{aligned}
$$

The u's are the displacements from the equilibrium positions, and $\hat{\mathbf{r}}$ is a unit vector connecting the equilibrium positions. The Ni atoms were placed at bulk positions with a nearestneighbor distance of $0.2489 \mathrm{~nm}$. The parameters $\lambda_{i j}$ and $\mu_{i j}$ were fitted on the elastic constants ${ }^{35}$ and cell parameters ${ }^{36}$ of the bulk. The values of all parameters are given in Table I.

\section{B. Simulation model}

The surface is modeled by a slab consisting of four layers of eight times eight Ni atoms. Periodic boundary conditions have been used in the lateral direction for the $\mathrm{Ni}-\mathrm{Ni}$ interactions. The methane molecule has interactions with the $64 \mathrm{Ni}$ atoms in the top layer of the slab. The surface temperature is set according to the following procedure. The Ni atoms are placed in equilibrium positions and are given random velocities out of a Maxwell-Boltzmann distribution with twice the surface temperature. The velocities are corrected such that the total momentum of all surface atoms is zero in all directions, which fixes the surface in space. Next the surface is allowed to relax for $350 \mathrm{fs}$. We do the following ten times iteratively. If at the end of previous relaxation the total kinetic energy is above or below the given surface temperature, then all velocities are scaled down or up with a factor of $\sqrt{1} .1$, respectively. Afterward a new relaxation simulation is performed. The end of each relaxation run is used as the beginning condition of the surface for the actual scattering simulation.

The initial perpendicular carbon position was chosen $180 \mathrm{~nm}$ above the equilibrium $z$-position of the top layer atoms and was given randomly parallel $(x, y)$ positions within the central surface unit cell of the simulation slab for the normal incidence simulations. The methane was placed in a random orientation with the bonds and angles of the methane in the minimum of the gas phase potential. The initial rotational angular momentum was generated randomly from a Maxwell-Boltzmann distribution for the given nozzle temperature for all three rotation axis separately. No vibrational kinetic energy was given initially. Initial translational velocity was given to all methane atoms according to the translational energy. The simulations under an angle were
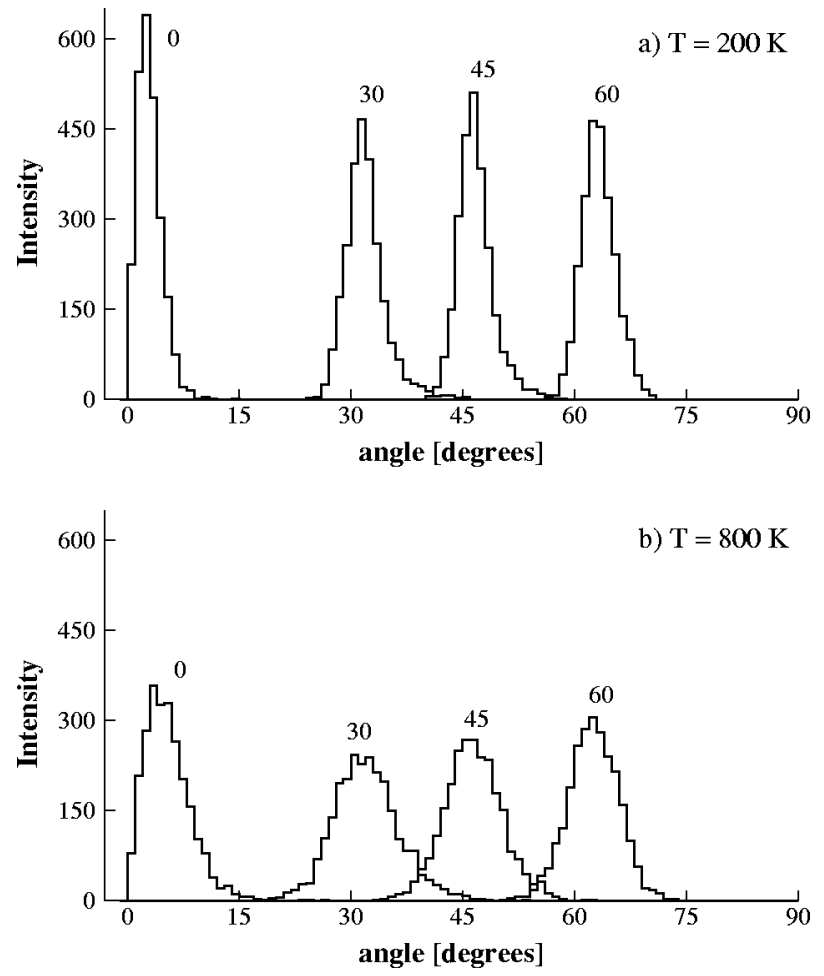

FIG. 1. The distribution of the scattering angle for a total initial translational energy of $96 \mathrm{~kJ} / \mathrm{mol}$ with incidence angles of $0^{\circ}, 30^{\circ}, 45^{\circ}$, and $60^{\circ}$ with the surface normal. Both the nozzle and surface temperature are (a) $200 \mathrm{~K}$ and (b) $800 \mathrm{~K}$.

given parallel momentum in the [110] direction. The parallel positions have been translated according to the parallel velocities in such a way that the first collision occurs one unit cell before the central unit cell of the simulation box. We tested other directions, but did not see any differences for the scattering.

Each scattering simulation consisted of 2500 trajectories with a simulation time of $1500 \mathrm{fs}$ each. We calculated the (change of) translational, total kinetic, rotational and vibrational kinetic, intramolecular potential, and total energy of the methane molecule, and the scattering angles at the end of each trajectory. We calculated for them the averages and standard deviations, which gives the spread for the set of trajectories, and correlations coefficients from which we can abstract information about the energy transfer processes.

\section{RESULTS AND DISCUSSION}

We will now present and discuss the results of our simulations. We begin with the scattering angle distribution. Next we will explain this in terms of the energy dissipation processes. Finally we will compare our simulation with previous theoretical and experimental scattering studies, and discuss the possible effects on the dissociation of methane on transition metal surfaces.

\section{A. Scattering angles}

Figure 1 shows the scattering angle distribution for different incidence angles with an initial total translational energy of $96 \mathrm{~kJ} / \mathrm{mol}$ at nozzle and surface temperatures of both 
200 and $800 \mathrm{~K}$. The scatter angle is calculated from the ratio between the normal and the total parallel momentum of the whole methane molecule. We observe that most of the trajectories scatter some degrees toward the surface from the specular. This means that there is relatively more parallel momentum than normal momentum at the end of the simulation compared with the initial ratio. This ratio change of the non-normal incidence scattering angles is almost completely caused by a decrease of normal momentum.

The higher nozzle and surface temperatures have almost no influence on the peak position of the distribution, but give a broader distribution. The standard deviation in the scattering angle distribution goes up from $2.7^{\circ}, 2.4^{\circ}$, and $2.2^{\circ}$ at $200 \mathrm{~K}$ to $4.4^{\circ}, 3.8^{\circ}$, and $3.4^{\circ}$ at $800 \mathrm{~K}$ for incidence angles of $30^{\circ}, 45^{\circ}$, and $60^{\circ}$, respectively. This means that the angular width is very narrow, because the full width at half maximum (FWHM) are usually larger than $20^{\circ} .{ }^{37}$ (The FWHM is approximately somewhat more than twice the standard deviation.) The broadening is caused almost completely by raising the surface temperature, and has again primarily an effect on the spread of the normal momentum of the molecule. This indicates that the scattering of methane from $\mathrm{Ni}(111)$ is dominated by a thermal roughening process.

We do not observe an average out-of-plane diffraction for the non-normal incidence simulations, but we do see some small out-of-plane broadening. The standard deviations in the out-of-plane angle were $0.9^{\circ}, 1.8^{\circ}, 3.4^{\circ}$ at a surface temperature of $200 \mathrm{~K}$, and $1.7^{\circ}, 3.3^{\circ}$, and $6.0^{\circ}$ at $800 \mathrm{~K}$ for incidence angles of $30^{\circ}, 45^{\circ}$, and $60^{\circ}$ with the surface normal. Raising the (rotational) nozzle temperature has hardly any effect on the out-of-plane broadening.

\section{B. Energy dissipation processes}

\section{Translational energy}

Figure 2 shows the average energy change of some energy components of the methane molecule between the end and the beginning of the trajectories as a function of the initial total translational energy. The incoming angle for all is $0^{\circ}$ (normal incidence), and both the nozzle and surface are initially $400 \mathrm{~K}$. If we plot the normal incidence translational energy component of the simulation at $96 \mathrm{~kJ} / \mathrm{mol}$ for the different incidence angles, then we see a similar relation. This means that there is normal translational energy scaling for the scattering process in general, except for some small differences discussed later on.

Most of the initial energy of methane is available as translational energy, so it cannot be surprising that we see here the highest energy loss. The translational energy loss takes a higher percentage of the initial translational energy at higher initial translational energies. Since almost all of the momentum loss is in the normal direction, we also see that the loss of translational energy can be found back in the normal component of the translational energy for the nonnormal incidence simulations.

The average change of the total energy of the methane molecule is less negative than the average change in translational energy, which means that rotational and vibrational

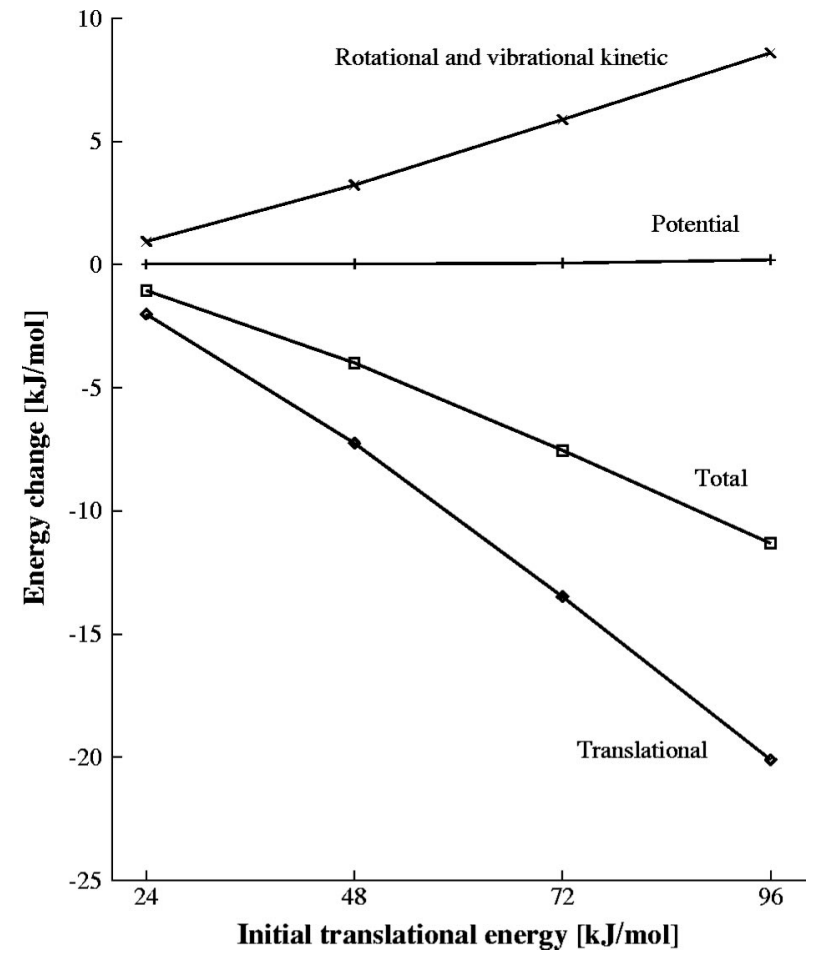

FIG. 2. The average energy change $(\mathrm{kJ} / \mathrm{mol})$ of the methane translational energy, the methane total energy, the methane potential energy, and the methane rotational and vibrational kinetic energy as a function translational kinetic energy $(\mathrm{kJ} / \mathrm{mol})$ at normal incidence. The nozzle and surface temperature were $400 \mathrm{~K}$.

excitation do not account for all the translational energy loss. So there has to be a net transfer of the initial methane energy toward the surface during the scattering, which is somewhat more than half of the loss of translational energy. The percentage of transferred energy to the surface related to the normal incidence translational energy is also enhanced at higher incidence energies. There is somewhat more translational energy loss, and energy transfer toward the surface for the larger scattering angles, than occurs at the comparable normal translational energy at normal incidence. This is caused probably by interactions with more surface atoms, when the molecule scatters under a larger angle with the surface normal.

In Fig. 2 we also plotted the average change of methane potential energy and the change of rotational and vibrational kinetic energy of methane. We observe that there is extremely little energy transfer toward the potential energy, and a lot of energy transfer toward rotational and vibrational kinetic energy. Vibrational motion gives an increase of both potential and kinetic energy. Rotational motion gives only an increase in kinetic energy. So this means that there is almost no vibrational inelastic scattering, and very much rotational inelastic scattering.

Figure 3 shows the standard deviations in the energy change of some energy components of methane versus the initial translational energy at normal incidence for a nozzle and surface temperature of $200 \mathrm{~K}$. (The temperature effects will be discussed below.) The standard deviations in the energy changes are quite large compared to the average values. The standard deviations in the change of the methane trans- 


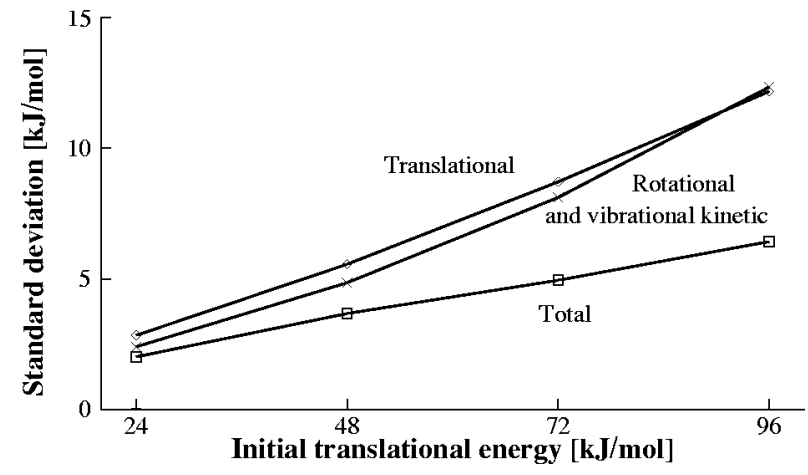

FIG. 3. The standard deviation in the energy change $(\mathrm{kJ} / \mathrm{mol})$ of the methane translational energy, the methane total energy, and the methane rotational and vibrational kinetic energy as a function of the initial translational energy $(\mathrm{kJ} / \mathrm{mol})$ at normal incidence. The surface and nozzle temperature are both $200 \mathrm{~K}$.

lational energy and in the change of methane rotational and vibrational kinetic energy increase more than the standard deviation in the change of methane total energy, when the initial translational energy is increased. We also observe that the internal energy gain and translational energy loss are stronger correlated at higher normal translational energies, because the correlation coefficients are $0.72,0.76,0.83$, and 0.87 at initial normal translational energies of $24,48,72$, and $96 \mathrm{~kJ} / \mathrm{mol}$, respectively, at a nozzle and surface temperature of $200 \mathrm{~K}$.

We find again an identical relation if we plot the standard deviations versus the initial normal energy component of the scattering at different incidence angles. The standard deviations are much smaller in the parallel than in the normal component of the translational energy, so again only the normal component of the translational energy is important. Although the standard deviations in the translational energy is smaller at larger incidence angles than at smaller incidence angles, we see in Fig. 1 that the spread in the angle distribution is almost the same. This is caused by the fact that at large angles deviations in the normal direction has more effect on the deviation in the angle than at smaller angles with the normal.

\section{Surface temperature}

An increase of surface temperature gives a small reduction of average translational energy loss (around 5\% from $200 \mathrm{~K}$ to $800 \mathrm{~K}$ at $96 \mathrm{~kJ} / \mathrm{mol}$ normal incidence). This is the reason why we do not observe a large shift of the peak position of the scattering angle distribution. However, an increase of surface temperature does have a larger effect on the average energy transfer to the surface, but this is in part compensated through a decrease of energy transfer to rotational energy.

Figure 4 shows the standard deviations in the energy change of the translational energy, the methane total energy, and the methane rotational and vibrational kinetic energy as a function of the surface temperature. We observe that the standard deviation in the change of rotational and vibrational kinetic energy hardly changes at increasing surface temperature. At a low surface temperature it is much higher than the

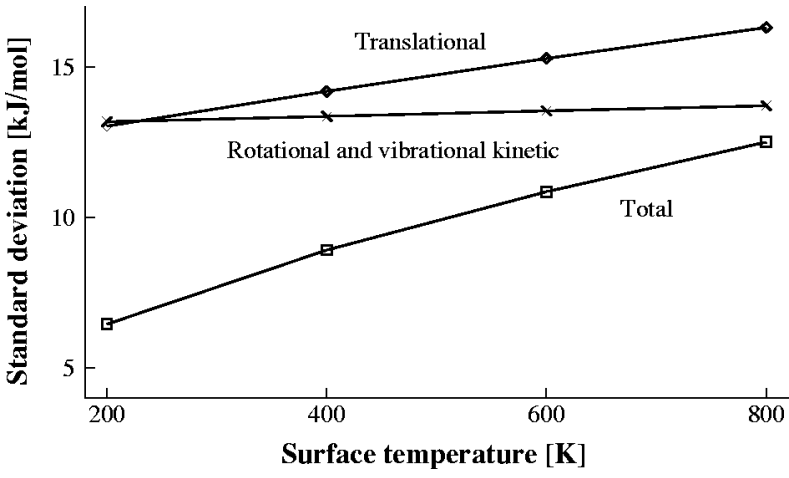

FIG. 4. The standard deviation in the energy change $(\mathrm{kJ} / \mathrm{mol})$ of the methane translational energy, the methane total energy, and the methane rotational and vibrational kinetic energy as a function of the surface temperature (K). The nozzle temperature is $400 \mathrm{~K}$, and the translational energy is 96 $\mathrm{kJ} / \mathrm{mol}$ at normal incidence.

standard deviation in the change of the methane total energy. So the standard deviation in the change of translational energy is mainly caused by the transfer of translational to rotational energy. The standard deviation in the change of the methane total energy increases much at higher surface temperature. This results also in an increase of the standard deviation in the change of translational energy, which means that the surface temperature influences the energy transfer process between translational and surface motion. The spread in the change of translational energy is related to the spread in the scattering angle distributions. It is now clear that the observed broadening of the scattering angle distribution with increasing surface temperature is really caused by a thermal roughening process.

\section{Nozzle temperature}

Figure 5 shows the dependency of the standard deviations for the different energy changes on the nozzle temperature. From this figure it is clear that the nozzle temperature has relative little influence on the standard deviations in the different energy changes. Therefore we observe almost no peak broadening in the scattering angle distribution due to

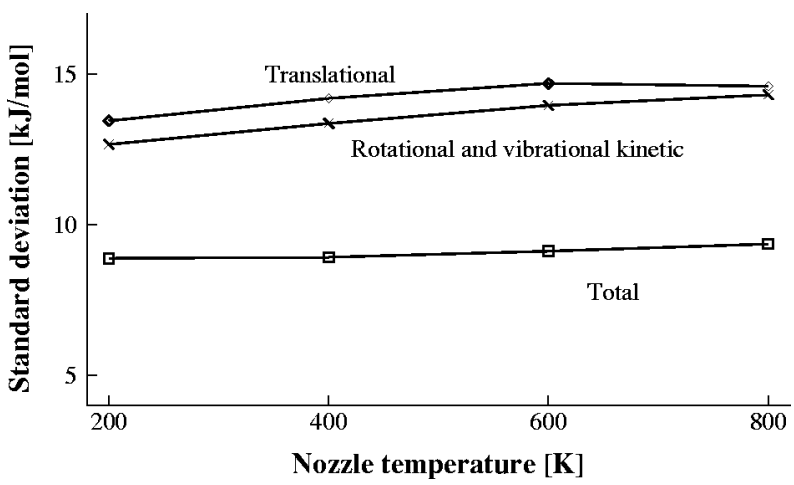

FIG. 5. The standard deviation in the energy change $(\mathrm{kJ} / \mathrm{mol})$ of the methane translational energy, the methane total energy, and the methane rotational and vibrational kinetic energy as a function of the nozzle temperature (K). The surface temperature is $400 \mathrm{~K}$, and the translational energy is 96 $\mathrm{kJ} / \mathrm{mol}$ at normal incidence. 
the nozzle temperature. The nozzle temperature has also no influence on the average change of rotational and vibrational kinetic energy, which means that this part of the energy transfer process is driven primarily by normal incidence translational energy.

We have to keep in mind that we only studied the rotational heating by the nozzle temperature, and that we did not take vibrational excitation by nozzle heating into account. From our wave packet simulations we know that vibrational excitations can contribute to a strong enhancement of vibrational inelastic scattering. ${ }^{23}$ So the actual effect of raising the nozzle temperature can be different than sketched here.

\section{Comparison with other studies}

\section{Scattering angles and the Hard Cube Model}

The angular dependence of scattered intensity for a fixed total scattering angle has only been measured at $\mathrm{Pt}(111){ }^{26,27}$ The measurement has been compared with the predictions of the Hard Cube Model (HCM) as described in Ref. 31. There seems to be more or less agreement for low translational energies under an angle around $45^{\circ}$ with the surface, but is anomalous at a translational energy of $55 \mathrm{~kJ} / \mathrm{mol}$. The anomalous behavior has been explained by altering the inelastic collision dynamics through intermediate methyl fragments.

Although our simulations are for $\mathrm{Ni}(111)$ instead of $\operatorname{Pt}(111)$ and we calculate real angular distributions, we will show now that the HCM is insufficient for describing the processes involved with the scattering of methane in our simulation. The HCM neglects the energy transfer to rotational excitations, and overestimates the energy transfer to the surface. This is not surprising, because the HCM is constructed as a simple classical model for the scattering of gas atoms from a solid surface. The basic assumptions are that (1) the interaction of the gas atom with a surface atom is represented by an impulsive force of repulsion, (2) the gassurface intermolecular potential is uniform in the plane of the surface, (3) the surface is represented by a set of independent particles confined by square well potentials, (4) the surface particles have a Maxwellian velocity distribution. ${ }^{31}$ Assumption (1) excludes inelastic rotational scattering, because the gas particle is an atom without moment of inertia. So the HCM misses a large part of inelastic scattering. However, it still predicts scattering angles much more below the incidence angles than we found from our simulation. For example, the HCM predicts an average scattering angle with the surface normal of $64^{\circ}$ from $\mathrm{Ni}(111)$, at an incidence angle of $45^{\circ}$ at a surface temperature four times lower than the gas temperature. This is much more than for $\operatorname{Pt}(111)$, because the mass ratio between the gas particle and the surface atom is higher for $\mathrm{Ni}(111)$. There are several explanations for this error. First, assumption (3) is unreasonable for atomic surfaces with low atom weight, because the surface atoms are strongly bound to each other. This means that effectively the surface has a higher mass than assumed. ${ }^{38} \mathrm{Sec}-$ ond, there is no one-on-one interaction between surface atom and methane molecule, but multiple hydrogen atoms interacting with different $\mathrm{Ni}$ atoms. Third, the methane molecule is not rigid in contrast to assumption (1). We have followed the energy distribution during the simulation for some trajectories and find that the methane molecule adsorbs initial rotational and translational energy as vibrational energy in its bonds and bond angles when close to the surface, which is returned after the methane moves away from it.

It would be nice to test our model with molecular beam experiment of the scattering angles on surfaces with relatively low atom weight, which also try to look at rotational inelastic scattering.

\section{Wave packet simulations}

Let us now compare the full classical dynamics with our fixed oriented wave packet simulations, ${ }^{21-23}$ because this was initially the reason to perform the classical dynamics simulations. Again we observe very little vibrational inelastic scattering. This is in agreement with the observations in the time-of-flight experiments on $\mathrm{Pt}(111){ }^{28,29}$

Since we used our wave packet simulations to deduce consequences for the dissociation of methane, we have to wonder whether the observed inelastic scattering in our classical simulations changes the picture of the dissociation in our previous publications. Therefore we have to look at what happens at the surface. We did so by following some trajectories in time.

We find approximately the same energy rearrangements for the classical simulations as discussed for the wave packet simulations for the vibrational groundstate in Refs. 22 and 23. Again most of the normal translational energy is transferred to the potential energy terms of the surface repulsion [see Eq. (1)]. This repulsive potential energy was only given back to translational energy in the wave packet simulations, because the orientations and surface were fixed. For the classical trajectory simulations presented in this article, the repulsive potential energy is transferred to translational, rotational, and surface energy through the inherent force of the repulsive energy terms. We observe almost no energy transfers to translational energy parallel to the surface, so exclusion of these translational coordinates in the wave packet simulations do not effect our deduction on the dissociation. The energy transfers to the rotational and surface energy during the collision make it harder for the molecule to approach the surface. This will have a quantitative effect on the effective bond lengthening near the surface, but not a qualitative.

The remaining problem deals with the effect of rotational motion on the dissociation probability and steering. Our first intention was to look for the favorable orientation at the surface, but from following some trajectories it is clear that steering does not seem to occur. There is always some rotational motion, and the molecule leaves the surface often with another hydrogen pointing toward the surface than when it approaches the surface. This indicates that multiple bonds have a chance to dissociate during one collision. However, it will be very speculative to draw more conclusions on the dissociation of methane based on the scattering in these classical trajectory simulations. Classical trajectory simulation with an extension of our potentials with an exit channel for dissociation can possibly teach us more. 


\section{CONCLUSIONS}

We have performed classical dynamics simulations of the rotational vibrational scattering of nonrigid methane from a corrugated $\mathrm{Ni}(111)$ surface. Energy dissipation and scattering angles have been studied as a function of the translational kinetic energy, the incidence angle, the (rotational) nozzle temperature, and the surface temperature.

We find the peak of the scattering angle distribution somewhat below the incidence angle of $30^{\circ}, 45^{\circ}$, and $60^{\circ}$ at a translational energy of $96 \mathrm{~kJ} / \mathrm{mol}$. This is caused by an average energy loss in the normal component of the translational energy. An increase of initial normal translational energy gives an enhancement of inelastic scattering. The energy loss is transferred for somewhat more than half to the surface and the rest mostly to rotational motion. The vibrational scattering is almost completely elastic.

The broadening of the scattering angle distribution is mainly caused by the energy transfer process of translational energy to rotational energy. Heating of the nozzle temperature gives no peak broadening. Heating of the surface temperature gives an extra peak broadening through thermal roughening of the surface.

The Hard Cube Model seems to be insufficient for describing the scattering angles of methane from $\mathrm{Ni}(111)$, if we compare its assumptions with the processes found in our simulations.

\section{ACKNOWLEDGMENTS}

This research has been financially supported by the Council for Chemical Sciences of the Netherlands Organization for Scientific Research (CW-NWO), and has been performed under the auspices of the Netherlands Institute for Catalysis Research (NIOK).

${ }^{1}$ J. H. Larsen and I. Chorkendorff, Surf. Sci. Rep. 35, 163 (2000).

${ }^{2}$ C. T. Rettner, H. E. Pfnür, and D. J. Auerbach, Phys. Rev. Lett. 54, 2716 (1985).

${ }^{3}$ C. T. Rettner, H. E. Pfnür, and D. J. Auerbach, J. Chem. Phys. 84, 4163 (1986).

${ }^{4}$ M. B. Lee, Q. Y. Yang, and S. T. Ceyer, J. Chem. Phys. 87, 2724 (1987).

${ }^{5}$ P. M. Holmbad, J. H. Larsen, and I. Chorkendorff, J. Chem. Phys. 104, 7289 (1996).
${ }^{6}$ A. V. Hamza and R. J. Madix, Surf. Sci. 179, 25 (1987).

${ }^{7}$ P. M. Holmbad, J. Wambach, and I. Chorkendorff, J. Chem. Phys. 102, 8255 (1995).

${ }^{8}$ A. C. Luntz and D. S. Bethune, J. Chem. Phys. 90, 1274 (1989).

${ }^{9}$ A. V. Walker and D. A. King, Phys. Rev. Lett. 82, 5156 (1999).

${ }^{10}$ A. V. Walker and D. A. King, J. Chem. Phys. 112, 4739 (2000).

${ }^{11}$ D. C. Seets, M. C. Wheeler, and C. B. Mullins, J. Chem. Phys. 107, 3986 (1997).

${ }^{12}$ D. C. Seets, C. T. Reeves, B. A. Ferguson, M. C. Wheeler, and C. B. Mullins, J. Chem. Phys. 107, 10229 (1997).

${ }^{13}$ J. H. Larsen, P. M. Holmblad, and I. Chorkendorff, J. Chem. Phys. 110, 2637 (1999).

${ }^{14}$ L. B. F. Juurlink, P. R. McCabe, R. R. Smith, C. L. DiCologero, and A. L. Utz, Phys. Rev. Lett. 83, 868 (1999).

${ }^{15}$ J. Harris, J. Simon, A. C. Luntz, C. B. Mullins, and C. T. Rettner, Phys. Rev. Lett. 67, 652 (1991)

${ }^{16}$ A. C. Luntz and J. Harris, Surf. Sci. 258, 397 (1991).

${ }^{17}$ A. C. Luntz and J. Harris, J. Vac. Sci. Technol. A 10, 2292 (1992).

${ }^{18}$ A. C. Luntz, J. Chem. Phys. 102, 8264 (1995).

${ }^{19}$ A. P. J. Jansen and H. Burghgraef, Surf. Sci. 344, 149 (1995).

${ }^{20}$ M.-N. Carré and B. Jackson, J. Chem. Phys. 108, 3722 (1998).

${ }^{21}$ R. Milot and A. P. J. Jansen, J. Chem. Phys. 109, 1966 (1998).

${ }^{22}$ R. Milot and A. P. J. Jansen, Surf. Sci. 452, 179 (2000).

${ }^{23}$ R. Milot and A. P. J. Jansen, Phys. Rev. B 61, 15657 (2000).

${ }^{24}$ H. Asada, Jpn. J. Appl. Phys. 20, 527 (1981).

${ }^{25}$ H. Asada and T. Matsui, Jpn. J. Appl. Phys. 21, 259 (1982).

${ }^{26}$ S. Yagyu, Y. Kino, K. Ozeki, and S. Yamamoto, Surf. Sci. 433-435, 779 (1999).

${ }^{27}$ S. Yagyu, Y. Kino, T. Ikeuchi, T. Hiraoka, T. Kondo, and S. Yamamoto, Jpn. J. Appl. Phys. 38, 6910 (1999).

${ }^{28}$ S. Yagyu, T. Hiraoka, Y. Kino, and S. Yamamoto, Appl. Surf. Sci. 165, 217 (2000).

${ }^{29}$ T. Hiraoka, S. Yagyu, T. Kondo, T. Ikeuchi, and S. Yamamoto, Jpn. J. Appl. Phys. 39, 612 (2000).

${ }^{30}$ T. Andersson, F. Althoff, P. Linde, M. Hassel, M. Persson, and S. Andersson, J. Chem. Phys. 113, 9262 (2000).

${ }^{31}$ R. M. Logan and R. E. Stickney, J. Chem. Phys. 44, 195 (1966).

${ }^{32}$ The NAG Fortran Library Manual Mark 17, 1st ed., NAG Ltd., Oxford, 1995, routine D02CJF.

${ }^{33}$ D. L. Gray and A. G. Robiette, Mol. Phys. 37, 1901 (1979).

${ }^{34}$ T. J. Lee, J. M. L. Martin, and P. R. Taylor, J. Chem. Phys. 102, 254 (1995).

${ }^{35}$ Landolt-Börnstein, Vol. II of NS III, edited by K.-H. Hellwege and A. M. Hellwege (Springer, Berlin, 1986).

${ }^{36}$ N. W. Ashcroft and N. D. Mermin, Solid State Physics (Holt, Rinehart and Winston, New York, 1976).

${ }^{37}$ A. E. Wiskerke and A. W. Kleyn, J. Phys.: Condens. Matter 7, 5195 (1995).

${ }^{38}$ E. K. Grimmelmann, J. C. Tully, and M. J. Cardillo, J. Chem. Phys. 72, 1039 (1980) 